Review

\title{
Therapeutic Angiogenesis for Treating Cardiovascular Diseases
}

\author{
Lorenzo Devezai, 2, Jeffrey $\mathrm{Choi}^{3}$, Fan Yang',4,凹 \\ 1. School of Medicine, Stanford University, Stanford, CA, 94305, USA; \\ 2. Department of Bioengineering, Stanford University, Stanford, CA, 94305, USA; \\ 3. Department of Biology, Stanford University, Stanford, CA, 94305, USA; \\ 4. Department of Orthopaedic Surgery, Stanford University, Stanford, CA, 94305, USA.
}

Corresponding author: Prof. Fan Yang, Department of Orthopaedic Surgery and Bioengineering, Stanford University School of Medicine, 300 Pasteur Dr., Edwards R105, Stanford, CA, 94305-5341. E-mail: fanyang@stanford.edu Tel.: 650-725-7128 Fax: 650-723-9370.

(C) Ivyspring International Publisher. This is an open-access article distributed under the terms of the Creative Commons License (http://creativecommons.org/ licenses/by-nc-nd/3.0/). Reproduction is permitted for personal, noncommercial use, provided that the article is in whole, unmodified, and properly cited.

Received: 2012.03.29; Accepted: 2012.05.29; Published: 2012.08.16

\begin{abstract}
Cardiovascular disease is the leading cause of death worldwide and is often associated with partial or full occlusion of the blood vessel network in the affected organs. Restoring blood supply is critical for the successful treatment of cardiovascular diseases. Therapeutic angiogenesis provides a valuable tool for treating cardiovascular diseases by stimulating the growth of new blood vessels from pre-existing vessels. In this review, we discuss strategies developed for therapeutic angiogenesis using single or combinations of biological signals, cells and polymeric biomaterials. Compared to direct delivery of growth factors or cells alone, polymeric biomaterials provide a three-dimensional drug-releasing depot that is capable of facilitating temporally and spatially controlled release. Biomimetic signals can also be incorporated into polymeric scaffolds to allow environmentally-responsive or cell-triggered release of biological signals for targeted angiogenesis. Recent progress in exploiting genetically engineered stem cells and endogenous cell homing mechanisms for therapeutic angiogenesis is also discussed.
\end{abstract}

Key words: Angiogenesis, Cardiovascular Diseases

\section{Clinical significance}

Cardiovascular disease (CVD) represents a global medical and economic problem with high morbidity and mortality rates. The World Health Organization (WHO) has listed CVD as the number one cause of death worldwide. In the United States alone, CVD accounted for $32.8 \%$ of the approximately 2.5 million deaths in 2008 [1]. The prevalence of CVD is similarly staggering, with an estimated 82.6 million American adults ( 1 in 3 ) having one or more types of CVD. Of these, 40.4 million are estimated to be older than 60 years of age with the average annual rates of first time CVD events increasing with age [1]. For those that have experienced one CVD event, CVD has been listed as one of the 15 leading conditions that cause functional disability, thereby affecting quality of life and the ability to work.

Depending on the organs affected, CVD can be classified into coronary artery disease, cerebrovascular disease, peripheral arterial disease and aortic (thoracic or abdominal) atherosclerosis. CVD is generally characterized by narrowing or occlusion of the blood supply of these vascular beds, and is most commonly caused by atherosclerosis [2-3]. Treatment options for CVD generally aim to re-establish blood flow through the affected vascular beds, and are administered based on the severity of the disease $[2,4]$. For early-stage disease, management focuses on lifestyle modifications to reduce the number of modifia- 
ble risk factors. As the disease progresses, pharmacological or surgical interventions may be needed to increase the blood flow through the affected tissue or to reduce the energy requirements. Pharmacological therapy acts to decrease oxygen demand by applying drugs to decrease the heart rate; or to increase the blood supply by applying drugs that cause vascular smooth muscle dilation. In cases of acute disease or full vascular occlusion, vascular stents may be used to expand vessels when one or a few vessels are affected, while surgical bypass is necessary when multiple vascular beds are occluded [4]. Despite the set of currently available treatment options for patients with CVD, there is a subset of patients with advanced disease for whom surgical revascularization is not an option due to the existence of various co-morbidities that prohibit them from undergoing surgical procedures.

Driven by the clinical demands, therapeutic angiogenesis aims to stimulate and augment the growth of new blood vessels from pre-existing vessels in order to re-supply blood flow to affected ischemic tissues. In this review, we discuss strategies developed for therapeutic angiogenesis, including direct delivery of angiogenic growth factors and the delivery of cells to ischemic tissues. Recent progress on therapeutic angiogenesis utilizing polymeric biomaterials, combined stem cell and gene therapy and regulation of endogenous stem cell homing are also discussed.

\section{Biology of angiogenesis}

There are several mechanisms by which blood vessel formation occurs including vasculogenesis, angiogenesis, and arteriogenesis [5-6]. Each of these processes is interrelated and leads to the formation of the vasculature of the body. The earliest blood vessel formation in a developing embryo arises via vasculogenesis, in which endothelial progenitor cells coalesce to form solid cords. These initially lumenless cords then transform into patent vessels in the process of tubulogenesis [6]. Unlike the de novo blood vessel formation process associated with vasculogenesis, angiogenesis is defined as sprouting new blood vessels from pre-existing blood vessel networks and is important for expanding the vascular bed initially formed via vasculogenesis. Arteriogenesis is the maturation of arterio-arteriolar anastomoses by the recruitment and coating of pre-formed vessels with pericytes or vascular smooth muscle cells. Arteriogenesis results in completely developed, functional arteries [7]. In the post-natal period, angiogenesis and arteriogenesis play the major role in re-vascularizing under-supplied tissues [5].

Under normal physiological conditions, there is a steady state in which quiescent endothelial cells are maintained by autocrine signaling including vascular endothelial growth factor (VEGF), NOTCH, angiopoietin-1 and fibroblast growth factor (FGF) [5-6]. This steady state may become disrupted under conditions of low oxygen, inflammation, wound healing, or within a tumor. Such hypoxic conditions can be sensed by endothelial cells and other stromal cells, which express oxygen sensors and hypoxia-inducible factors, such as prolyl hydroxylase domain 2 (PHD2) and hypoxia-inducible factor $2 a(H I F-2 a)$ [5-6]. When hypoxia is sensed, angiogenic signals are released, such as VEGF, angiopoietin-2, and FGF [5-6]. Initially, these signals cause pericytes to detach from the vessel walls and endothelial cells to loosen their cell-cell junctions, which enable the blood vessels to dilate. This increases vascular permeability and allows plasma proteins to extravasate and form a provisional extracellular matrix (ECM) through which ECs can migrate. Simultaneously, proteases liberate angiogenic molecules from the ECM, which further potentiate the process. The migrating ECs differentiate to become guiding tip cells or proliferating stalk cells. The tip cells lead the direction of sprouting, while the stalk cells proliferate to elongate the sprouting vessels. Blood flow is initiated when two growing vessels meet and fuse. Maturation later ensues as pericytes are stimulated to cover endothelial cells under the action of platelet-derived growth factor-B (PDGF-B), angiopoietin-1 (ANG-1), and transforming growth factor- $\beta$ (TGF- $\beta$ ) [5]. It is known that bone-marrow derived endothelial progenitor cells (EPCs) also participate in angiogenesis and become incorporated into the vascular wall, but their importance is not well understood [8].

\section{Therapeutic angiogenesis}

Therapeutic angiogenesis aims to induce, augment and control the host angiogenic response in order to re-vascularize ischemic tissues, and often involves delivery of growth factors or stem/progenitor cells. Growth factors may be delivered in the form of proteins or genes encoding target proteins. The premise behind this approach is to apply well-studied growth factors (VEGF, FGF) in ischemic tissues to guide angiogenic cellular and tissue behavior. Cell therapy may similarly act to induce the angiogenic response by the release of paracrine signals. Delivered cells may also act by becoming incorporated into the growing vascular supply, thereby acting as building blocks to form new blood vessels. Both mechanisms likely occur upon delivery of cells to ischemic tissues. Transmyocardial laser revascularization offers another strategy for therapeutic angiogenesis, which is 
believed to stimulate host angiogenic response by injuring the ischemic myocardium in specified locations [9].

\section{I. Growth factor therapy}

Various growth factors have been applied for therapeutic angiogenesis including VEGF, bFGF, hepatocyte growth factor (HGF), PDGF, ANG-1 and insulin-like growth factor (IGF-1). Among these, VEGF and bFGF are the most well-studied and have reached human clinical trials. VEGF is the most important regulator of physiological angiogenesis during growth, healing and in response to hypoxia $[5,10]$. VEGF is upregulated 30 -fold by hypoxia-inducible transcription factor, which is more than any other inducible angiogenic factor. However, when administered alone, VEGF may lead to the formation of leaky, unstable capillaries. PDGF-B can help stabilize nascent blood vessels by recruiting mesenchymal progenitors, and co-delivery of VEGF and PDGF has been shown to lead to early formation of mature vessels [11]. bFGF is among the first discovered angiogenic factors to have both angiogenic and arteriogenic properties, and FGF receptors are expressed on both endothelial cells and smooth muscle cells, which may facilitate formation of a mature blood vessel network $[5,10]$. The HGF family induces potent angiogenic responses by binding to the c-MET receptor, which is expressed on endothelial cells, vascular smooth muscle cells and hematopoietic stem cells. HGF is known to have mitogenic, angiogenic, anti-apoptotic, and anti-fibrotic activities in various cells [10].

To date, there have been several pre-clinical and clinical studies evaluating the safety and efficacy of growth factors for inducing angiogenesis in ischemic heart disease and peripheral arterial disease (PAD). Early phase I and II human clinical trials with VEGF, bFGF and HGF were promising, but larger randomized placebo-controlled trials failed to demonstrate significant benefits in the approved end-points. Preclinical data demonstrate that the delivery of bFGF or VEGF eventually results in unstable vessel growth that resembles immature tumor vasculature. The limited clinical response to growth factor therapy is likely due to a number of factors. Growth factor delivery is generally limited by their rapid diffusion, poor biostability and short half-lives in vivo. Furthermore, it often requires supraphysiological doses or multiple injections, which could lead to excessive uncontrolled vascular formation in undesired locations.

\subsection{Cell-based therapy}

Cell-based therapy is the most extensively studied approach so far for therapeutic angiogenesis and may contribute via either directly participating in new vessel formation or secreting paracrine signals. Excellent reviews have been published previously with detailed discussion on this topic [12-13]. In this review, we have chosen to focus on more recently developed platforms for therapeutic angiogenesis including biomaterials-based approach, combined stem cells and gene therapy and exploiting stem cell homing for angiogenesis. As a therapeutic approach, cells have the potential to act as hypoxia-responsive paracrine release vehicles by virtue of their diverse cytokine contents. As cells migrate and respond to the environment, they may also lead to more dynamic paracrine release. The most extensively studied cell populations for therapeutic angiogenesis are endothelial progenitor cells (EPC) and bone marrow-mesenchymal stem cells (BMMSCs). EPCs have been found to mobilize in response to ischemia and become incorporated into the growing vasculature $[8$, 14]. However, it should be recognized that various studies have employed different protocols for isolating this population of cells, and this has resulted in inconsistent expression profiles among different studies, which consists of true EPCs and other cell types, such as hematopoietic stem cells [12]. For this reason, this cell population has been reported and identified according to the method of cell isolation, e.g., "granulocyte colony stimulating factor (G-CSF) mobilized peripheral blood cells," or "bone marrow aspirate mononuclear cells" (BMNCs). These cell populations can differentiate into endothelial cells, pericytes, or smooth muscle cells, thereby acting as building blocks for angiogenesis, but may also potentiate vascular growth through paracrine mechanisms. BMMSCs also have the potential for differentiation into vascular support cells, but they more likely contribute to angiogenesis by the release of proangiogenic factors. Adipose-derived stem cells (ADSC) exhibit similar differentiation potential and paracrine release characteristics as BMMSCs, and is currently being evaluated in preclinical trials for therapeutic angiogenesis. Compared to BMMSC, ADSCs are more abundant and easier to isolate, yet more studies are still needed to fully assess their potential for therapeutic angiogenesis.

The main advantages of autologous stem cell therapy relate to immunogenicity and ethical issues. Because these cells will be transplanted back into the same patient from which they are harvested, there should be no immune rejection, such as that seen in allogeneic implants. Also, since these progenitor cells are isolated from adult tissues and are not derived from fetal tissues, they face no major identified ethical concerns. Furthermore, as adult stem cells, their dif- 
ferentiation potential is relatively limited with lesser risk for aberrant tissue formation and tumorigenesis. On the other hand, cell-based therapies still face several limitations. Upon isolation, the cell population is often heterogeneous, which may lead to varied responses. To obtain the large cell numbers needed for transplantation, ex vivo cell expansion is often required, which leads to regulatory concerns and increased cost and time, thereby increasing the barriers for clinical translation. Furthermore, the cell engraftment efficiency is typically very low upon transplantation, and may induce inflammatory responses. Still, cell-based therapies have met with success in the clinic. In a meta-analysis of cell therapy for PAD, it was found that autologous cell delivery (BMMSCs and G-CSF mobilized peripheral blood cells) led to improved indices of ischemia and subjective symptoms (pain-free walking) [15]. However, the study also pointed out limitations of their analysis, such as publication bias and the inclusion of unblinded study results. Further work is needed to determine the efficacy of autologous stem cell therapy.

\section{Polymeric biomaterials for therapeutic angiogenesis}

Polymeric biomaterials may serve as drug delivery depots for controlled release of biological signals in a temporal and spatial-controlled manner. In the ideal situation, such systems may release growth factor within a physiological range over the course of several days to weeks, thereby overcoming the issues of short protein half-lives and rapid diffusion from the site. Polymeric biomaterials have been applied in therapeutic angiogenesis to delay the release of single growth factors, to release multiple biologics or to achieve environment-responsive release of biologics in response to changes in $\mathrm{pH}$ or cell-mediated matrix metalloproteinase activities.

\section{I. Commonly utilized polymers for thera- peutic angiogenesis}

Polymers for therapeutic angiogenesis include synthetic-based polymers, such as poly(lactic-co-glycolic acid) (PLGA) and poly(ethylene glycol) (PEG), as well as naturally-derived polymers, such as alginate or gelatin. PLGA was among the first to be studied for drug release and many initial systems for therapeutic angiogenesis utilized PLGA as a base polymer for the controlled release of VEGF [16-18]. PLGA is a favorable polymer due to its biodegradability and ability to control release, but when degraded it increases the local acidity, which could cause undesirable inflammation. PEG is a biocompatible polymer that is inert by nature, with easily tunable biochemical and mechanical properties [19]. PEG has been utilized to create cell-responsive hydrogels for VEGF release [20]. Alginate and gelatin are composed of natural polymers that can interact well with cells in vivo and may also help to control drug release. However, natural-derived materials suffer from batch-to-batch variance, and they are less tunable in chemical properties compared to synthetic polymers.

\subsection{Strategies for modulating growth factor release using biomaterials}

To achieve effective therapeutic angiogenesis, it would be desirable to have a sustained release of biological signals over time. However, most drug delivery systems have an initial "burst-release," where the large majority of loaded growth factors is released in the first few hours. While the mechanisms underlying "burst-release" have not yet been entirely elucidated, several parameters are believed to play a critical role in regulating matrix-controlled drug release including processing conditions, surface characteristics, and geometry [21]. Several strategies have been developed to delay such burst release by modulating physical interactions, ionic/biochemical affinity interactions, and covalent binding between the loaded biologics and the polymeric biomaterials (Figure 1).

\subsection{Physical interactions}

Physical interactions or physical entrapment can delay protein release based on the size of the drug relative to the pore size of the scaffolds. Controlled release of VEGF from PLGA scaffolds improved angiogenesis compared to free-VEGF administration in a myocardial ischemia model or hindlimb ischemia model [17-18]. These reports demonstrate the benefit of prolonged release of VEGF over bolus growth factor injection. Biological signals can also be loaded into composite scaffolds using both PLGA microspheres containing alginate hydrogel, which also demonstrated statistically significant increases in vessel density compared to recombinant human-VEGF (rh-VEGF) injection alone in vivo [22].

\subsubsection{Ionic/biochemical interactions}

Proteins can form hydrogen or ionic bonds with macromolecules such as heparan sulfate. Such interactions can be utilized to slow down the protein release by incorporating heparin, heparan sulfate or hyaluronic acid into the drug-releasing scaffolds [23-27]. bFGF specifically binds to heparin and heparan sulfate, and loading bFGF into heparin conjugated PLGA nanospheres led to linear release up to 15 days [23]. This release can be further prolonged by encap- 
sulating these PLGA nanospheres into fibrin hydrogels [23]. Sustained release of bFGF resulted in significant improvements in capillary density and cell proliferation in a murine hindlimb ischemia model, [23]. Enhanced angiogenesis was also observed using heparin-containing chitosan hydrogels or fibrous matrices to release bFGF in vivo, and release can be tuned by varying heparin concentration in the scaffold [24, 27]. Heparin binding alginate hydrogels have also been developed for prolonged release of HGF, which resulted in significant increases in blood flow and capillary density compared to bolus HGF delivery [26]. These studies show that release of growth factor may be prolonged by exploiting heparin-binding interactions and this has potential for improving growth factor approaches for therapeutic angiogenesis.

\subsubsection{Covalent linking}

Lastly, some biologics can be covalently bound to the polymeric matrix via a hydrolytically degradable or MMP-degradable linker [20, 28-29]. It has been demonstrated that matrices incorporating covalently attached VEGF via a MMP-degradable linker can lead to the formation of a more controlled and stable vasculature [20, 29]. However, covalent linkage requires chemical modification of the biologic which can affect its biological activity. Also, the chemistry of the linker must be carefully designed to prevent unwanted immune responses [19].

\subsection{Co-delivery of multiple biologics}

Angiogenesis is a dynamic process that is regulated by complex biological signals. Polymeric biomaterials offer the potential to simultaneously or sequentially deliver multiple biologics better mimicking the complexity of angiogenesis [11, 30-35]. For example, VEGF is a potent angiogenic factor, but when delivered alone is not sufficient for developing a mature and stable vascular network. Dual delivery of VEGF and PDGF using PLGA or alginate hydrogel resulted in the for-

Figure I. Methods for incorporating biological signals into scaffolds for controlled release. A) Biologics encapsulated directly via physical entrapment. B) Biologics covalently tethered to polymer chains. C) Biologics loaded into microspheres and then encapsulated into hydrogel scaffolds. Reproduced with permission from ref [19].

mation of larger and more mature vessels and improved blood flow, thereby suggesting the potential benefits of co-delivering synergistic growth factors (Figure 2) [11, 30]. Biodegradable hydrogels such as hyaluronan (HA) has also been examined to achieve sequential delivery of VEGF and keratinocyte growth factor (KGF) [33]. When transplanted into the ear pinna of a mouse they found significant increases in microvascular density in the dual-delivery group from the HA hydrogel compared to dual growth factor bolus injections, or single growth factor delivery from HA hydrogels [33]. Gelatin-based hydrogel has also been shown as a promising depot for co-delivery of bFGF and G-CSF to enhance therapeutic angiogenesis [31]. bFGF is a mitogen and chemoattractant of both fibroblasts and endothelial cells, while G-CSF acts as a potent mobilizer of hematopoietic stem cells and bone marrow stromal cells. Dual release of bFGF and G-CSF from a gelatin hydrogel enabled increased reperfusion and capillary density by 2 weeks, which was sustained up to 8 weeks. In contrast, bFGF alone led to a reduction in these parameters by 8 weeks [31]. Bolus delivery of these factors led to improvements in capillary density, but no effects on blood reperfusion, which further supports the benefit of controlled release [31]. Dextran-based hydrogels capable of releasing multiple angiogenic factors (VEGF, SDF-1, IGF, Ang I) has been recently developed [35]. Significant increases in vessel number and size were observed when all four growth factors were delivered in a subcutaneous model compared to single or dual growth factor delivery, again suggesting the potential synergistic benefit of delivering multiple biological signals for therapeutic angiogenesis [35].

A

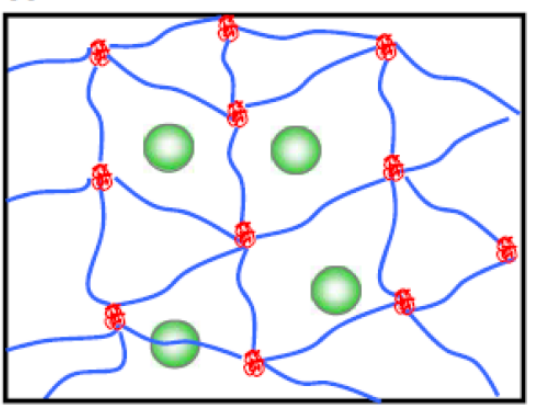

C

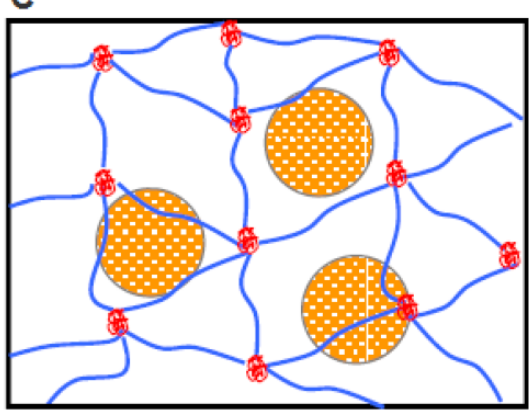

B

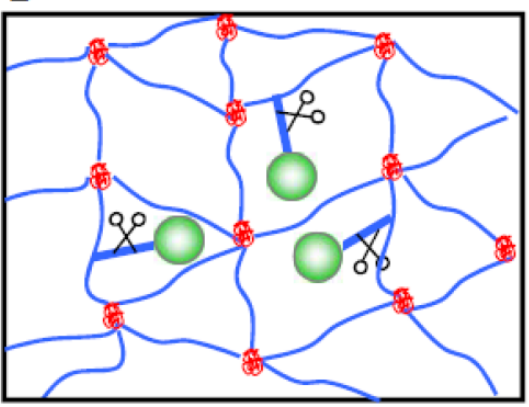

Dative therapeutics

Cleavable tether

Lo Proteases (enzymatic degradation) or water (hydrolytic degradation)

Therapeutics-loaded microparticle 
A

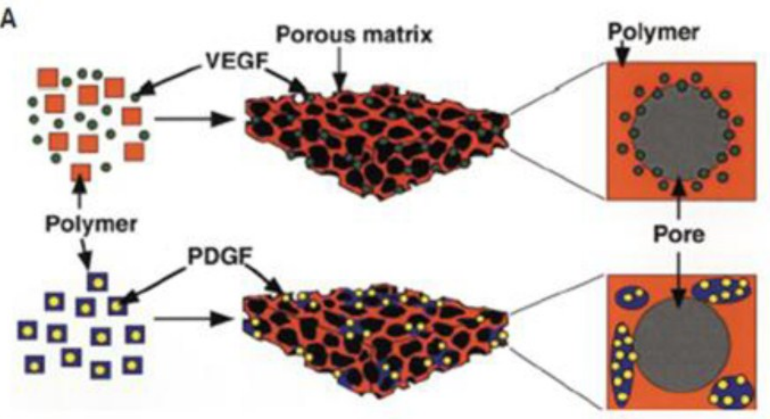

D

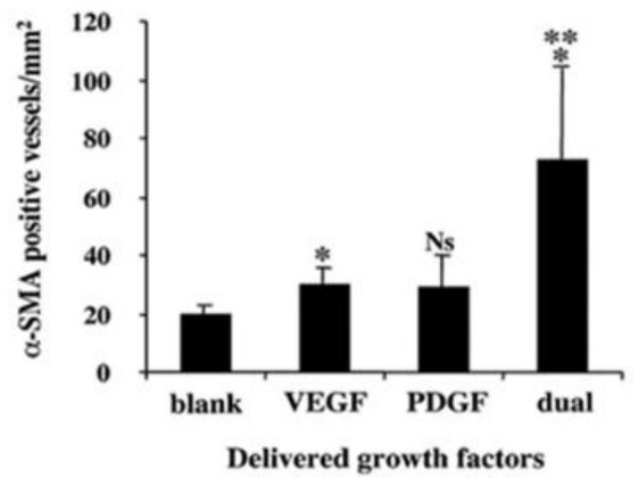

B
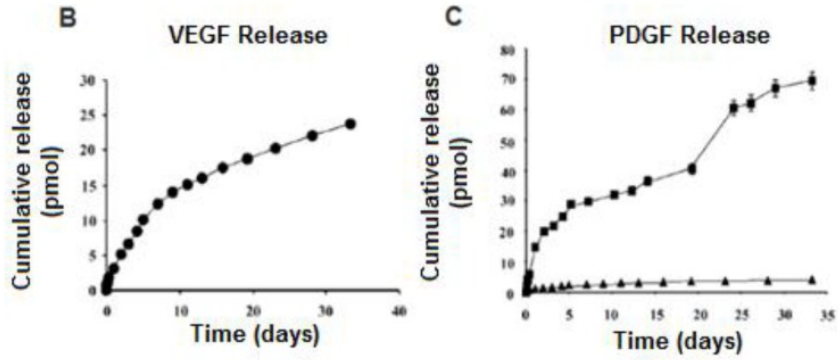

E

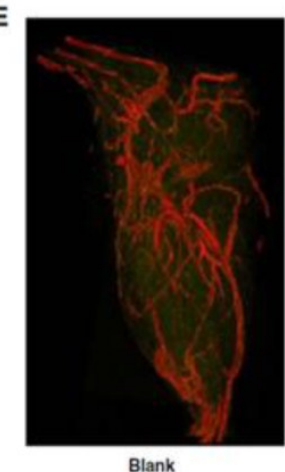

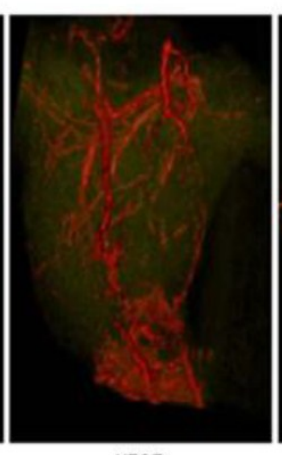

VEGF

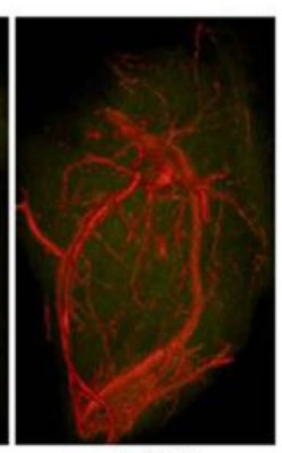

VEGFIPDGF

Figure 2. A) Polymeric scaffolds for dual delivery of VEGF and PDGF. (B) In vitro release kinetics of VEGF from scaffolds fabricated from PLGA (85:I5, lactide:glycolide), measured using I25I-labeled tracers. (C) In vitro release kinetics of PDGF pre-encapsulated in PLGA microspheres ( $\boldsymbol{A} 75: 25$, intrinsic viscosity $=0.69 \mathrm{dl} / \mathrm{g} ; 75: 25$, $\square$ intrinsic viscosity $=0.2 \mathrm{dl} / \mathrm{g}$ ), before scaffold fabrication. $\mathrm{D}$ ) Dual delivery led to early maturation of blood vessels as demonstrated by $\alpha$-smooth muscle actin staining at 2 weeks. E) Representative micro-CT images after 5 weeks intramuscular injections of alginate (Blank), alginate containing VEGFI65 (VEGF), or alginate containing VEGFI65 combined with PLGA microspheres containing PDGF-BB (VEGF/PDGF).(A-D) Reproduced with permission from ref [I I] (E) Reproduced with permission from ref [30].

\subsection{Temporal controlled release}

Angiogenic response to growth factors is time-dependent and the ability to control the temporal release of growth factors is highly desirable to achieve maximal benefits. In support of this concept, Silva and colleagues demonstrated that a gradual reduction in VEGF concentration led to increases in vessel sprouts in an in vitro sprout assay compared to consistently high VEGF concentration or increasing VEGF concentration over time (Figure 3) [36]. Tengood and colleagues developed a cellulose hollow fiber capable of sequential release of VEGF followed by sphingosine-1-phosphate (S1P) [34]. VEGF initiates angiogenesis by increasing vascular permeability and recruits endothelial cells, while S1P acts to stabilize intracellular junctions and decrease the permeability of endothelial cells but simultaneously inhibits endothelial cells migration [34]. If delivered together, these two signals may inhibit each other. Their results showed that a significant increase in angiogenic response was only observed when VEGF and S1P were delivered sequentially. In contrast, low angiogenic response was observed when the two factors were delivered simultaneously or if S1P was delivered first. These results demonstrate that growth factors can act in temporal manner depending on the stage of angiogenesis.

\subsection{Environmental-responsive hydrogels for releasing biologics}

To aid releasing angiogenic growth factors specifically in ischemic tissues, $\mathrm{pH}$ and temperature responsive hydrogels have been developed. $\mathrm{pH}$-responsive heparin functionalized chitosan/poly( $\gamma$-glutamic acid) nanoparticles have been developed to gel and enable sustained release of bFGF under low $\mathrm{pH}$, and then disintegrate and release heparin under normal physiological $\mathrm{pH}$ (Figure 4) [37]. This design aimed to utilize bFGF to induce angiogenesis, while releasing heparin as an anti-coagulant [37]. The efficacy of such a system for in vivo therapeutic angiogenesis remains unknown and requires further testing. Another study reported the development of a $\mathrm{pH}$ and temperature sensitive hydrogel capable of gelation at $\mathrm{pH}$ of 6.8 and at $37^{\circ} \mathrm{C}$ [38]. When transplanted in a rat model of myocardial ischemia, it led to statistically significant improve- 
ments in blood flow, capillary density and arteriole density compared to bolus injection and polymer only controls, and these parameters correlated with improvements in myocardial functions [38]. These re- sults demonstrate the feasibility and promise of applying environmental-responsive polymers to achieve better control over growth factor release.

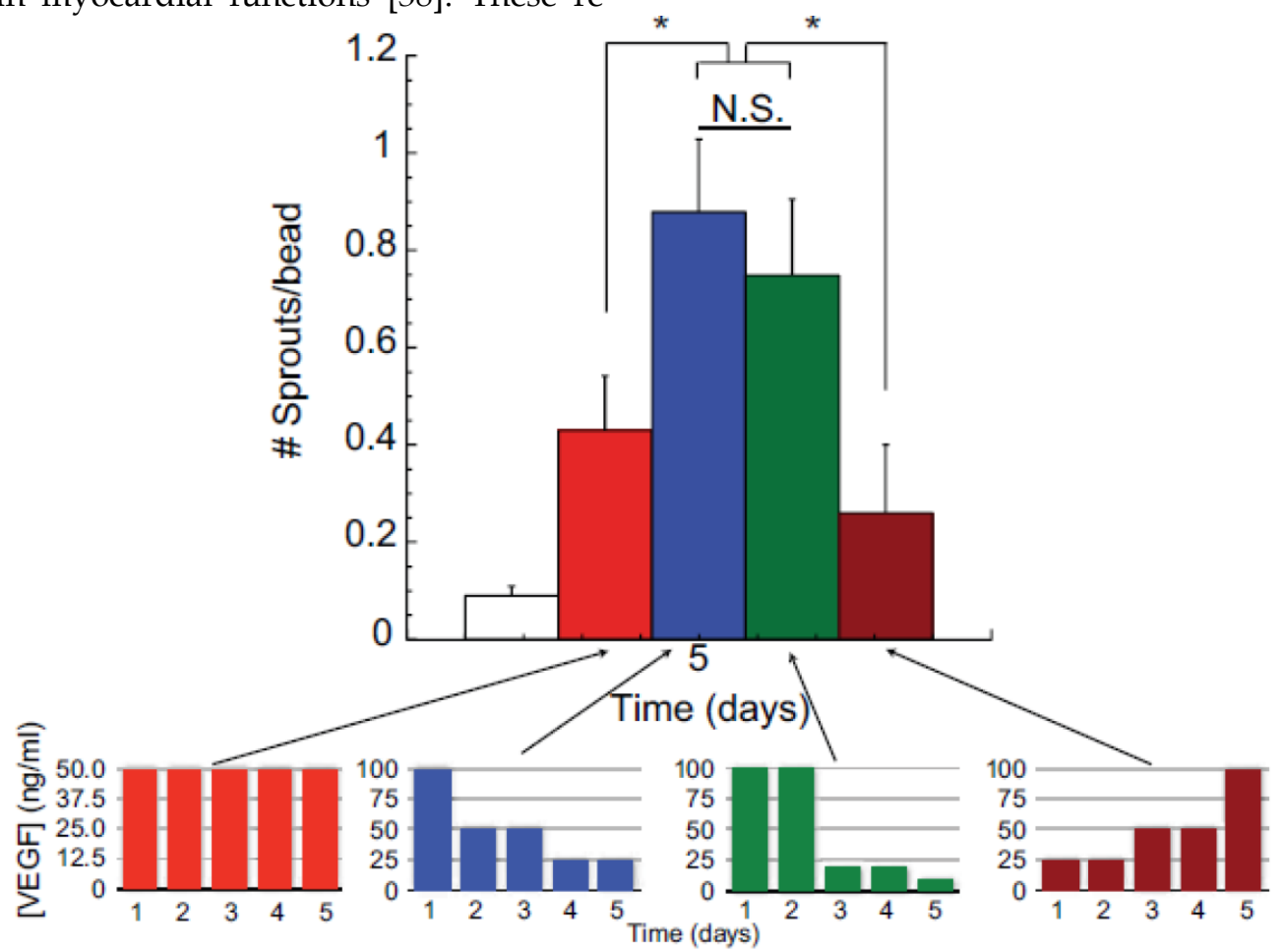

Figure 3. Effects of VEGF temporal presentation on angiogenesis. Varying amounts of VEGF is applied to human microvascular endothelial cells (HMVEC) over five days in a sprouting assay. Each color bar represents different temporal presentations of VEGF. Red bars represent the same concentration of VEGF applied daily over five days; blue bars represent decreasing VEGF dose over five days; green bars represent gradual decrease in VEGF concentration; and, brown bars represent increasing concentrations of VEGF applied. These are represented in the lower graphs. As shown in the larger graph, decreasing the VEGF dose from an initial high concentraion (blue bars) induced a greater number of endothelial cells sprouts, as compared to constant VEGF doses ( $50 \mathrm{ng} / \mathrm{ml}$ day) (red bars), increasing VEGF doses (brown bars), or a gradual VEGF dose decrease over time (green bars). Reproduced with permission from ref [36].
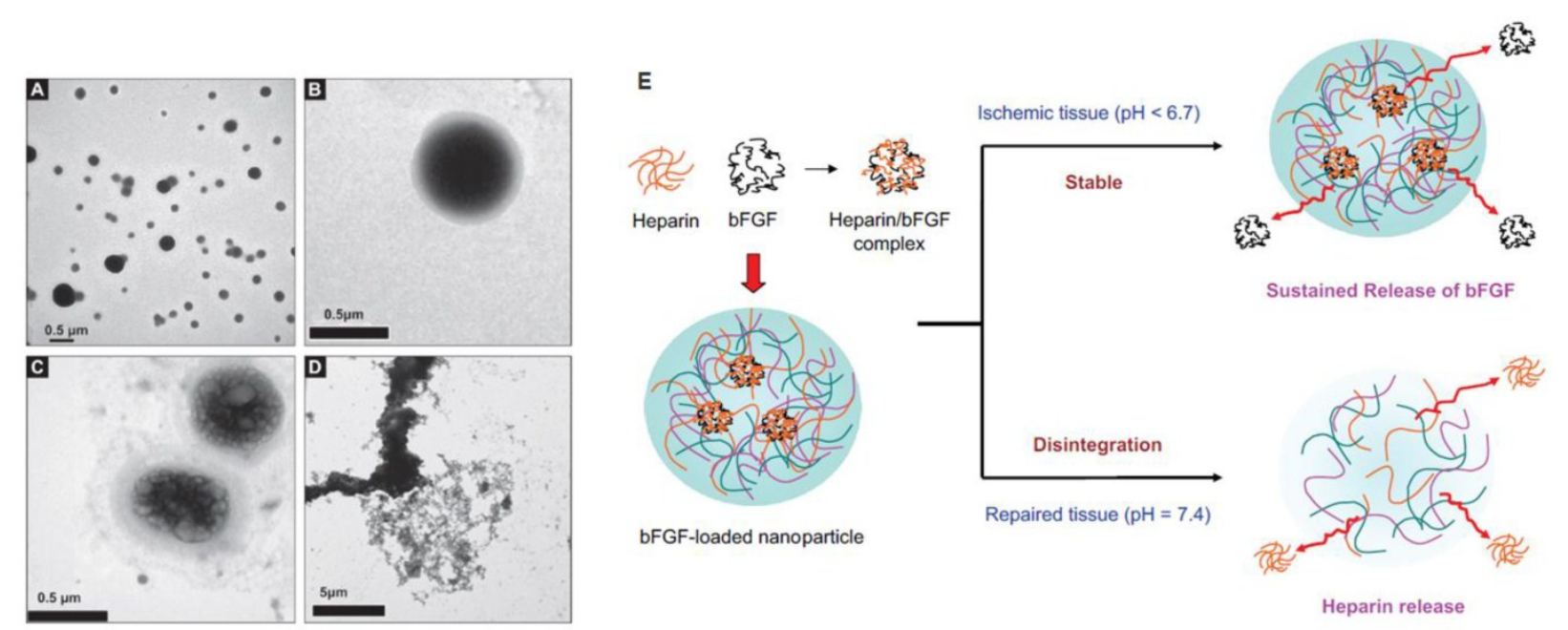

Figure 4. Environmental-responsive controlled release system for sustained bFGF delivery in ischemic tissue triggered by $\mathrm{pH}$. TEM micrography of heparinized chitosan/poly(gamma-glutamic acid) (HP-CS/g-PGA) nanoparticles at a distinct pH value: (A) pH 6.0 (after $2 \mathrm{~h}$ ), (B) $\mathrm{pH} 7.4$ (after $10 \mathrm{~min}$ ), (C) pH 7.4 (after $30 \mathrm{~min}$ ), (D) pH 7.4 (after $2 \mathrm{~h}$ ). (E) Release of bFGF or heparin from the smart nanoparticles, depending on the environmental $\mathrm{pH}$ variation. Reproduced with permission from ref [37]. 

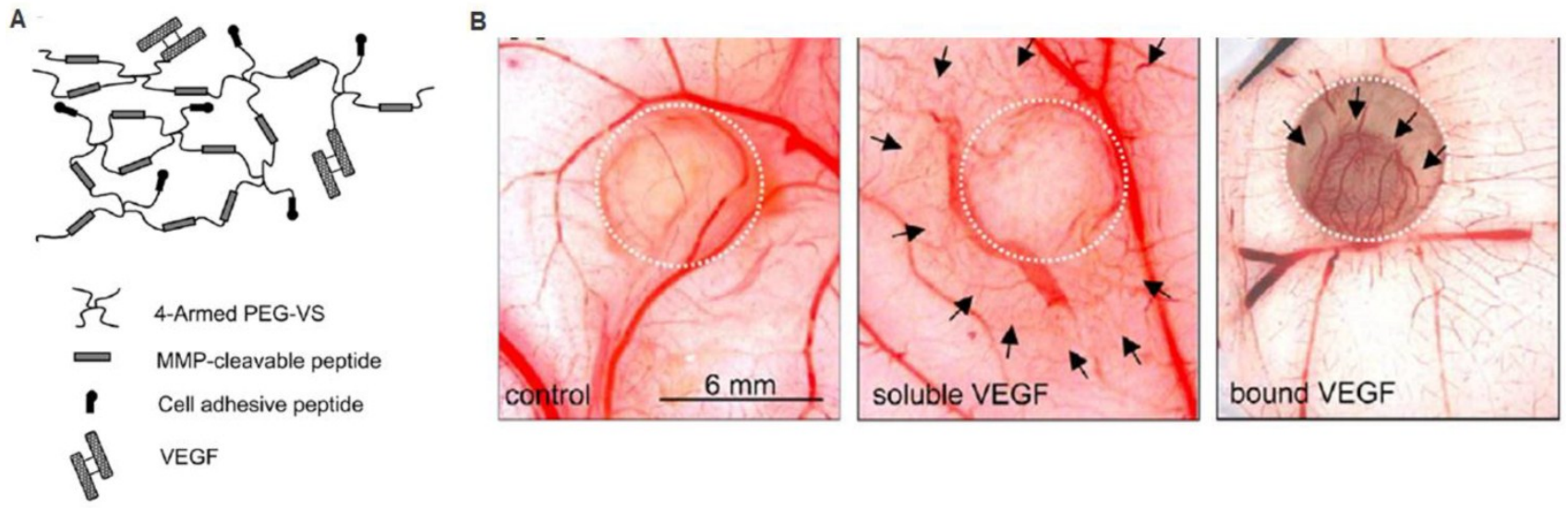

Figure 5. Cell-triggered VEGF delivery using biomimetic hydrogels. A) Schematic of MMP-degradable PEG hydrogels containing RGD peptide and covalently linked VEGF. B) Cell-triggered VEGF delivery led to targeted angiogenesis within the hydrogel region, whereas soluble VEGF treatment resulted in diffusive and uncontrolled angiogenesis. Reproduced with permission from ref [20].

\subsection{Biomimetic polymers to modulate cell response}

Biomimetic scaffolds containing cell-responsive domains can promote therapeutic angiogenesis. For example, matrices can be designed to interact with cells via integrin binding sites to facilitate cell migration and cell-cell interactions. Injecting RGD-modified alginate hydrogels into a chronic rat myocardial infarction model led to significant improvements in cardiac function 70 days post-infarction, with commensurate increases in arteriole density compared to non-RGD containing gel control [39]. In another study, an MMP-sensitive PEG hydrogel containing RGD and covalently linked VEGF was evaluated for therapeutic angiogenesis in vivo (Figure 5) [20]. The cell-responsive hydrogel led to angiogenesis specifically within the hydrogel, while soluble VEGF release led to increased vascular density in the surrounding tissue [20]. These systems demonstrate the importance of cell-matrix interactions and potential of exploring biomimetic polymers to modulate cell responses for therapeutic angiogenesis.

\section{Combined stem cell and gene-based therapy}

The therapeutic potential of stem cells can be further enhanced by combining with gene therapy. In this approach, stem cells can be genetically modified prior to transplantation in such a way that particular cellular processes are strategically exploited (Figure
6). In particular, researchers have focused on addressing the challenges associated with applying cells alone, such as insufficient paracrine release, poor cell survival upon engraftment, and lack of cell homing. Gene delivery can be used to over-express desired therapeutic factors to induce a biological response. In contrast to growth factor delivery, gene delivery may also be utilized to up-regulate expression of intracellular transcription factors or cell surface receptors. These strategies offer the advantage of controlling cell behavior at the intracellular signaling level.

\section{I. Viral vs. nonviral}

Both viral and non-viral vectors have been employed for gene delivery for therapeutic angiogenesis, with most studies utilizing a viral-based approach due to its high efficiency [40-50]. However, clinical translation of viral-based gene delivery is limited due to safety concerns such as immunogenicity and insertional mutagenesis. Non-viral methods provide a potentially safer alternative, and rely on physical methods such as electroporation and nucleofection, or using cationic lipids or polymers [51]. However, most non-viral based gene delivery methods suffer from low transfection efficiency and often high cytoxicity, and few studies for therapeutic angiogenesis have applied such methods [52-54]. All of the following genetic approaches described here utilized a viral-approach, except those described in the "Non-viral approaches" section. 
A

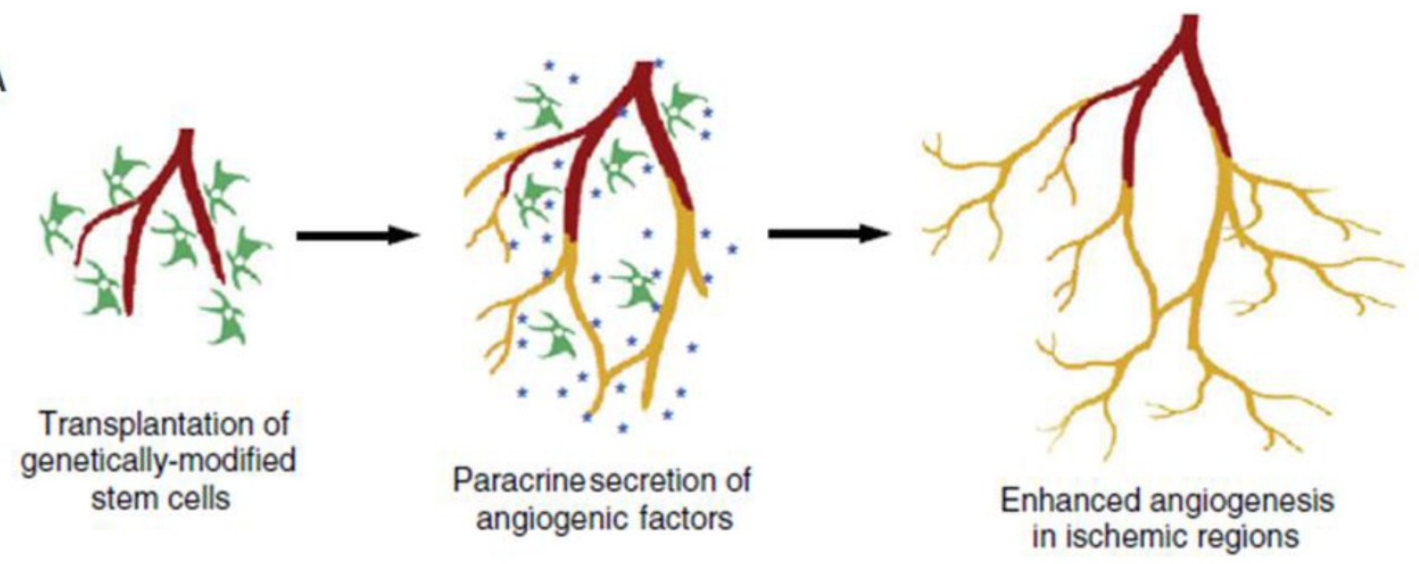

B
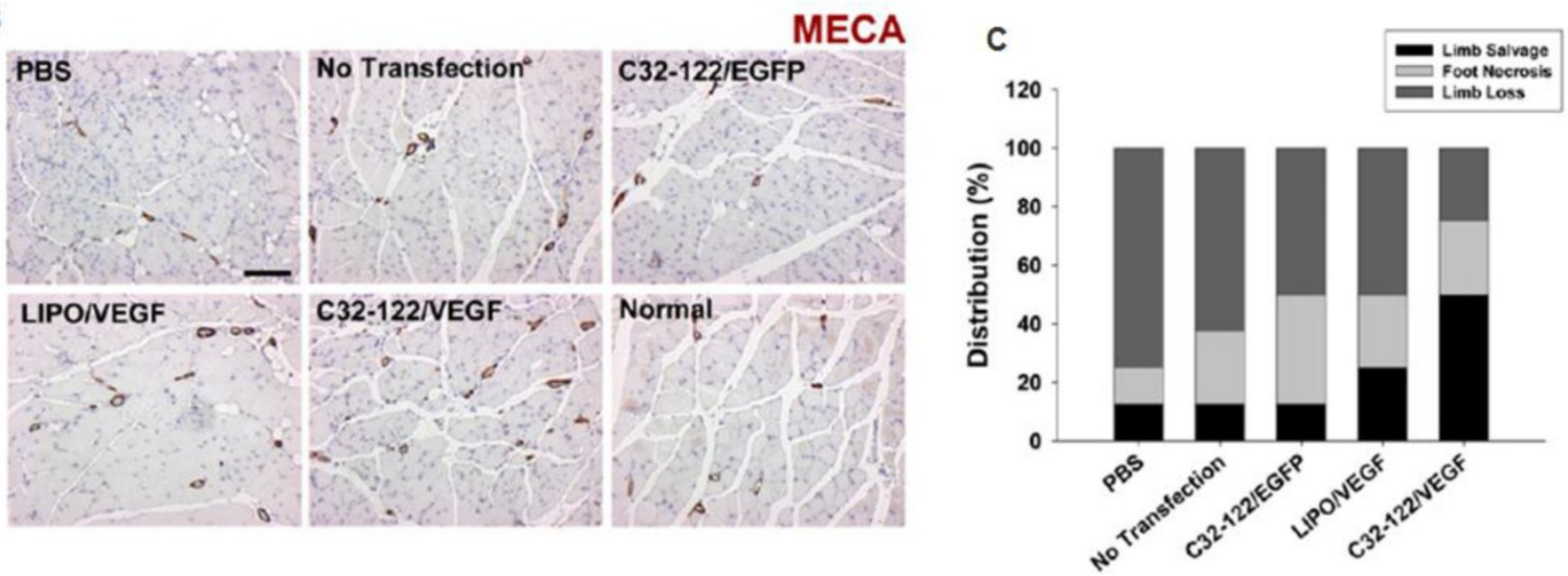

Figure 6. Combined stem cell and gene therapy approach for therapeutic angiogenesis (A). Transplantation of genetically modified stem cells into ischemic tissues led to enhanced paracrine secretion and angiogenesis in situ. Reproduced with permission from ref [5I] (B). VEGF-overexpressing MSCs using biodegradable polymeric nanoparticles led to enhanced angiogenesis and limb salvage in a murine hindlimb ischemia model (C). Reproduced with permission from ref [54].

\subsection{Stem cells overexpressing growth factors}

The efficacy of stem cell-based therapy may be augmented by overexpressing desirable paracrine signals that promote angiogenesis. Overexpressing VEGF reduced the number of EPCs needed by 30 -fold to achieve a similar therapeutic effect as unmodified EPCs alone in promoting angiogenesis and limb salvage [42]. By enhancing paracrine effects, fewer cells would be necessary to achieve therapeutic effects. Similarly, virally transduced BMMSCs overexpressing VEGF or ANG I led to significantly enhanced angiogenesis and improved cardiac function in a rat myocardial infarction model compared to non-transfected controls [43-44]. HGF and bFGF are known to stimulate both endothelial cells and smooth muscle cells, and may stimulate both the initiation and maturation of angiogenesis. Virally transduced MSCs or ADSCs overexpressing HGF resulted in improvement in cardiac performance and increasing number of capillaries $[41,50]$. The improvement in cardiac function can be detected as early as day 7 and continued over time, supporting the benefits of HGF overexpression on both early and late stages of angiogenesis. EPC overexpressing FGF1 also led to significant increases in mature vessel density by a-smooth muscle actin staining in vivo [40]. Overall, these studies demonstrate the potential of stem cells overexpressing angiogenic factors for accelerating blood vessel growth and tissue functions.

\subsection{Genetic modification to enhance cell sur- vival}

To enhance cell survival post-transplantion, ex vivo genetic modification of stem cells with cell survival factors has been studied [55-56]. Akt is a serine threonine kinase that is known to be involved in several cellular processes, such as glucose metabolism and cell migration. Foremost, among these processes is that it is a cell survival factor. Based on these effects, 
Mangi and colleagues sought to enhance survival of MSCs upon transplantation by overexpressing Akt1 by a retroviral method [55]. In comparison to GFP transfected controls, they found significant increases (over two-fold) in c-kit+ cells (transplanted cells) and decreases in c-kit+ cell apoptosis 24 hours post-transplantation, and this led to significant decreases in fibrosis and increases in cardiac function [55]. Their results also demonstrated improvements in cardiac function utilizing half the number of Akt-positive MSCs as LacZ controls $\left(2.5 \times 10^{5}\right.$ cells vs $5.0 \times 10^{5}$ cells) further supporting their hypothesis. Another report sought to build on these results by positing an interesting approach to co-overexpress a cell-survival factor with a growth factor. In this study, Ang1 and Akt co-overexpressing MSCs were developed utilizing adenovirus and then were transplanted into a rat myocardial infarction model. They demonstrated that Akt overexpression leads to enhanced cell engraftment as long as 3 months compared to non-transfected controls with resulting increases in mature vessel density and improvements in heart function [56]. However, it is unclear what the benefits of dual over-expression were, since the study lacked single Akt or Ang1 controls [56]. More work will need to be done to determine how these effects might synergize.

\subsection{Genetic modification to enhance stem cell homing}

Efficient cell homing to ischemic tissue is critical for achieving effective angiogenesis as the therapeutic effects are largely dependent upon the engraftment efficiency of transplanted cells. Current methods rely mostly on intramuscular or intravascular cell injection, which often results in poor cell engraftment at the site of ischemia. Gene therapy has been applied to modify stem cells to overexpress cell homing factors to enhance cell targeting to ischemic tissues [45, 47-49]. The hypoxia-inducible factor-1 (HIF-1)/stromal-derived factor-1 (SDF-1)/CXC receptor 4 (CXCR4) is a well-known axis that enables host cells to home to sites of ischemia [57]. Based on this understanding, both CXCR4 and SDF-1 have been studied for induction of transplanted cell homing and host cell recruitment [45, 47-49]. Two studies have reported the use of virally transduced CXCR4 overexpressing MSCs for therapeutic application in rat models of myocardial ischemia [45, 49]. The method of delivery was IV injection for both studies and each found significant increases in cell engraftment into the ischemic myocardium compared to non-transfected controls. However, these studies reported conflicting results with regards to increases in vessel density due to CXCR4 transduction in MSCs [45, 49]. This may be due to differences in negative controls with one demonstrating significant increases compared to EGFP-adenoviral transfected controls and one showing no difference compared to non-transfected controls. In either case, CXCR4-overexpressing MSCs led to improvements in cardiac function, thereby demonstrating that such increased cell homing may provide therapeutic effects by another mechanism. Tang and colleagues took another approach seeking to upregulate SDF-1 in MSCs for the purpose of recruiting host cells for therapeutic vascularization [47-48]. In their study, they chose to overexpress both VEGF and SDF-1 to achieve the effect of initiation and maturation of new blood vessels in a rat model of myocardial infarction [48]. Interestingly, they demonstrated that overexpression of SDF-1 leads to genetic upregulation of the survival factor, Akt [47-48]. They found that SDF-1 overexpression alone was able to mediate significant increases in vascular density by $\mathrm{vWF}$ and a-smooth muscle actin staining, but combination therapy led to significantly higher increases with corresponding improvements in cardiac function [48]. These approaches may become more interesting as an understanding of host cell recruitment for injury becomes better understood.

\subsection{Non-viral approaches}

Non-viral gene transfection approaches are important for clinical translation. However, few studies have reported the use of non-viral vectors for ex vivo gene modification in therapeutic angiogenesis. Many of the current approaches for non-viral gene transfer are unsatisfactory due to low transfection efficiency or high cytotoxicty. To overcome some of these issues, Yang and colleagues developed VEGF over-expressing MSCs utilizing novel non-viral biodegradable polymeric nanoparticles called poly( $\beta$-amino esters) that were able to achieve 4-5 times the transfection efficiency of the leading commercially available reagent, Lipofectamine 2000, with minimal cytoxicity [54]. When transplanted into a murine model of hindlimb ischemia, they found enhancement in angiogenesis and limb salvage compared to GFP transfected controls, thereby demonstrating the clinical potential of non-viral engineered stem cell-based therapy for therapeutic angiogenesis (Figure 6) [54]. In another study, a novel plasmid for a hypoxia inducible VEGF was transfected into MSCs utilizing a non-degradable polymer (linear poly-ethyliminine), which also demonstrated improved efficiency and cytoxicity characteristics compared to Lipofectamine. This plasmid was designed to enable better control over VEGF release, and led to 
improved cell engraftment, capillary density and increased cardiac functions in a rat myocardial ischemia model. These results demonstrate the promise of applying non-viral based gene delivery for therapeutic efficacy, which may provide a safer alternative than the viral approach.

\section{Exploiting stem cell homing for thera- peutic angiogenesis}

Stem cell therapy holds great potential for therapeutic angiogenesis, but in general, clinical translation of stem cell-based approaches for therapeutic angiogenesis has been slow due to several inherent limitations. First, most methods require lengthy procedures which include cell isolation, in vitro cell modification, and subsequent transplantation back into the patient. Such multi-step procedures lead to high cost and significant regulatory concerns. Second, the vast majority $(>90 \%)$ of transplanted cells die and few manage to migrate to the ischemic site. Third, ex vivo stem cell modifications may be dependent on culture conditions and be transient, and may thus lose effectiveness upon transplantation in vivo.

One strategy to overcome the aforementioned limitations is to exploit natural cell homing mechanisms to attract host progenitor cells to sites of ischemic injury. In the normal physiological state, bone marrow-derived populations are recruited to sites of hypoxia to participate in the angiogenic process [8, 58]. However, during states of cardiovascular disease, host cells often have reduced functionality and a compromised cell niche [58]. Various researchers have proposed methods to increase cell homing to ischemic tissue for therapeutic angiogenesis and for tissue engineering [58-59]. The number of circulating EPCs from the bone marrow has been shown to increase in response to ischemia [8], which led to improved corneal neovascularization when ischemia was induced in the mouse hindlimb, and applying cell mobilization factor GM-CSF led to further increases in angiogenesis. These results demonstrate that ischemia induced at a distant site can have an effect on angiogenesis throughout the body by cell mobilization [8]. The number of EPCs recruited to the site of ischemia has been shown to be correlated to the level of hypoxia [57]. Increasing hypoxia led to increasing SDF-1 expression and vessel density, which was attenuated using antibodies to block the SDF-1/CXCR4 pathway
[57]. Further study revealed that G-CSF induced mobilization of bone marrow-derived cells by disrupting the SDF-1/CXCR4 interactions in the bone marrow niche, with gradual decrease in SDF-1 signaling and increased CXCR4 expression over 5 days [60]. When G-CSF mobilized cells were transplanted into a bone marrow transplant model, cell engraftment was inhibited by blocking SDF-1 or CXCR4 pathway with antibodies. In a later study, it was shown that HSCs, EPCs and MSCs could be differentially mobilized by application of different combinations of G-CSF, CXCR4 antagonists and VEGF [61].

The potential of cell homing strategies for treating cardiovascular disease has been demonstrated by the application of G-CSF in a mouse model of myocardial ischemia. In a study performed by Orlic and colleagues, G-CSF was applied once daily for 5 days prior to inducing myocardial infarction, followed by 3 more days of G-CSF injection post-myocardial infarction [62]. This protocol resulted in over $70 \%$ mouse survival in comparison to about $20 \%$ mouse survival in the untreated group [62]. This treatment also led to increased left ventricular wall thickness and enhanced ejection fraction [62]. However, in practice, this type of protocol is unrealistic since myocardial infarction gives few early warning signs. Despite some positive results in animal models, clinical trials with G-CSF for acute myocardial infarction have been inconclusive. One potential cause for the unsatisfactory results may be the lack of homing efficiency of G-CSF mobilized cells to sites of injury due to the rapid cleavage of SDF-1 by CD26/dipeptidylpeptidase-IV (DPP-IV) [63]. By applying both G-CSF and DPP-IV inhibitor (Diprotin-A), researchers have shown significant increases in homing to the infarcted myocardium, which correlated with improvements in capillary density and cardiac function and mouse survival compared to G-CSF treatment alone (Figure 7) [63]. Since these factors are administered at the time of injury, this therapy may be more realistic for clinical intervention. The field of exploiting stem cell homing for therapeutic angiogenesis is young, and represents a promising future direction for therapeutic angiogenesis. For future work, polymeric scaffolds that are capable of in situ recruiting and programming endogenous stem cells in ischemic sites would provide valuable tools for reducing the translational barrier for therapeutic angiogenesis. 

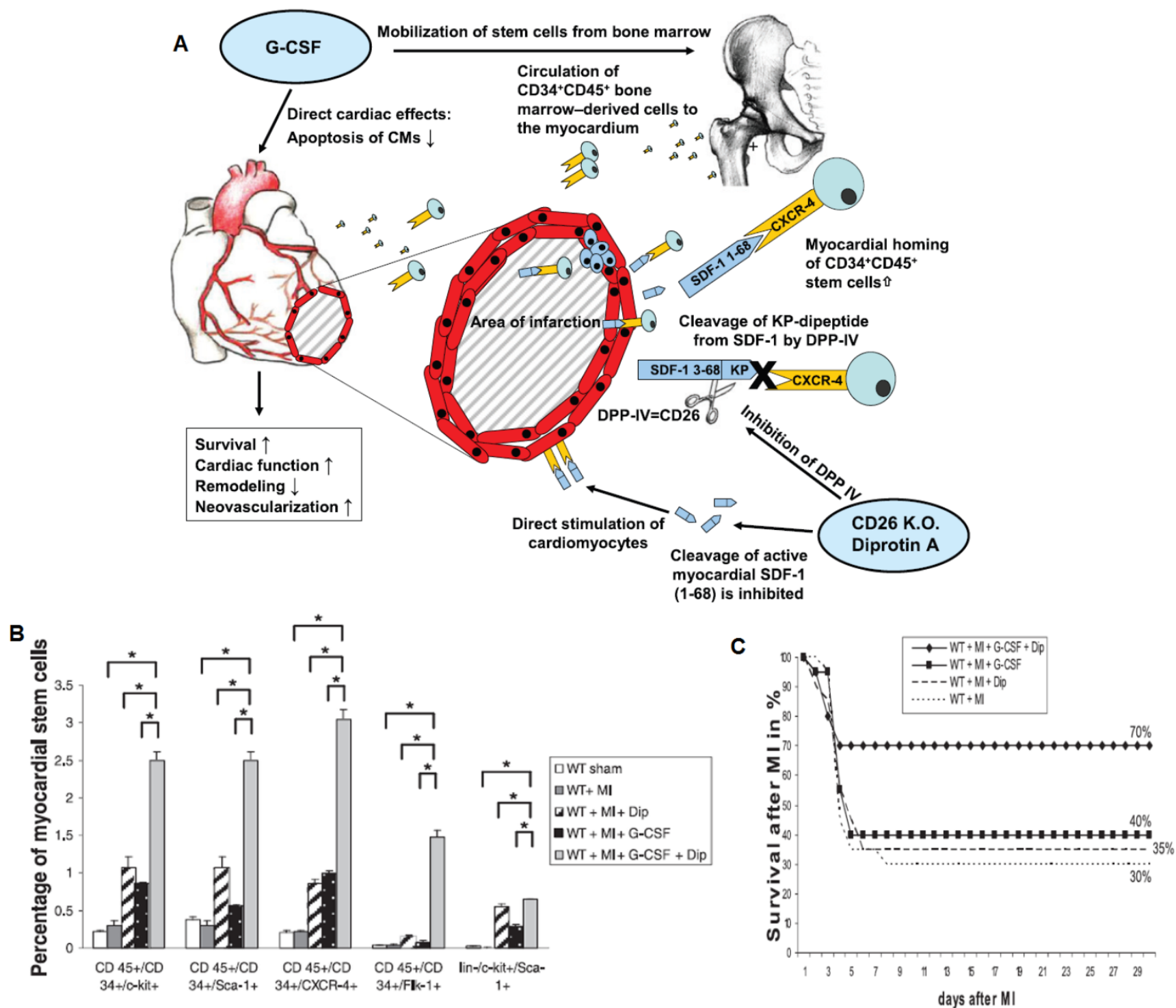

Figure 7. Exploiting cell homing for treating acute myocardial infarction. A) Signaling pathway related to stem cell homing to ischemic tissue. G-CSF promoted cell mobilization and Diprotin A (Dip) inhibited SDF-I protease, thereby maintaining the stability of cell homing factor SDF-I released from ischemic tissue. B) G-CSF and Dip dual treatment led to significantly increased myocardial stem cells detected in the ischemic tissue. C) G-CSF and Dip dual treatment increased mouse survival after myocardial infarction. Reproduced with permission from ref [63].

\section{Conclusions}

Therapeutic angiogenesis is an important interventional approach for treating a broad range of cardiovascular diseases, which represents a global challenge with high morbidity. Conventional strategies involve delivering growth factors or stem cells, or a combination of both. Polymeric biomaterials have the potential to enhance therapeutic angiogenesis by prolonging the release of angiogenic signals, and co-delivery of synergistic signals may further accelerate the formation of functional and mature blood vessel networks. Engineering biomimetic scaffolds that can release biological signals in an environmental-responsive manner would facilitate targeted angiogenesis, while mitigating the undesirable side effects caused by uncontrolled diffusion. Controlled release systems with precise temporal and spatial control will be of great value for therapeutic angiogenesis by mimicking the dynamic biological release profiles characteristic of normal angiogenesis processes. Combined stem cell and gene-based therapy may further improve the efficacy of using autologous cells alone by overexpressing therapeutic factors or cell homing factors, and its translation relies largely on safe and effective methods to deliver genes into 
stem cells. Finally, strategies exploiting cell homing may provide a solution to reduce the translational barrier of cell-based therapeutic angiogenesis by reducing the time and cost associated with ex vivo cell expansion and manipulation.

\section{Competing interests}

The authors have declared that no competing interest exists.

\section{References}

1. Roger V.L, et al. Heart disease and stroke statistics--2012 update: a report from the American Heart Association. Circulation, 2012. 125(1): e2-e220.

2. Libby $\mathrm{P}$ and Theroux P. Pathophysiology of coronary artery disease. Circulation, 2005. 111(25): 3481-8.

3. Jawad E and Arora R. Chronic stable angina pectoris. Dis Mon, 2008. 54(9): 671-89.

4. McFalls E.O, et al. Coronary-artery revascularization before elective major vascular surgery. N Engl J Med, 2004. 351(27): 2795-804.

5. Carmeliet P and Jain R.K. Molecular mechanisms and clinical applications of angiogenesis. Nature, 2011. 473(7347): 298-307.

6. $\mathrm{Xu} \mathrm{K}$ and Cleaver $\mathrm{O}$. Tubulogenesis during blood vessel formation. Semin Cell Dev Biol, 2011. 22(9): 993-1004.

7. Heil $\mathrm{M}$, et al. Arteriogenesis versus angiogenesis: similarities and differences. J Cell Mol Med, 2006. 10(1): 45-55.

8. Takahashi T, et al. Ischemia- and cytokine-induced mobilization of bone marrow-derived endothelial progenitor cells for neovascularization. Nat Med, 1999. 5(4): 434-8.

9. Horvath K.A. Transmyocardial laser revascularization. J Card Surg, 2008. 23(3): 266-76.

10. Losordo D.W and Dimmeler S. Therapeutic angiogenesis and vasculogenesis for ischemic disease. Part I: angiogenic cytokines. Circulation, 2004. 109(21): 2487-91.

11. Richardson T.P, et al. Polymeric system for dual growth factor delivery. Nat Biotechnol, 2001. 19(11): 1029-34.

12. Leeper N.J, Hunter A.L, and Cooke J.P. Stem cell therapy for vascular regeneration: adult, embryonic, and induced pluripotent stem cells. Circulation, 2010. 122(5): 517-26.

13. Rafii $S$ and Lyden D. Therapeutic stem and progenitor cell transplantation for organ vascularization and regeneration. Nat Med, 2003. 9(6): 702-12.

14. Asahara T, et al. VEGF contributes to postnatal neovascularization by mobilizing bone marrow-derived endothelial progenitor cells. EMBO J, 1999. 18(14): 3964-72.

15. Fadini G.P, Agostini C, and Avogaro A. Autologous stem cell therapy for peripheral arterial disease meta-analysis and systematic review of the literature. Atherosclerosis, 2010. 209(1): 10-7.

16. Cleland J.L, et al. Development of poly-(D,L-lactide--coglycolide) microsphere formulations containing recombinant human vascular endothelial growth factor to promote local angiogenesis. J Control Release, 2001. 72(1-3): 13-24.

17. Formiga F.R, et al. Sustained release of VEGF through PLGA microparticles improves vasculogenesis and tissue remodeling in an acute myocardial ischemia-reperfusion model. J Control Release, 2010. 147(1): 30-7.

18. Golub J.S, et al. Sustained VEGF delivery via PLGA nanoparticles promotes vascular growth. Am J Physiol Heart Circ Physiol, 2010. 298(6): H1959-65.

19. Lin C.C and Anseth K.S. PEG hydrogels for the controlled release of biomolecules in regenerative medicine. Pharm Res, 2009. 26(3): 631-43.

20. Zisch A.H, et al. Cell-demanded release of from synthetic VEGF, biointeractive cell ingrowth matrices for vascularized tissue growth. FASEB J, 2003. 17(15): 2260-2.

21. Huang $X$ and Brazel C.S. On the importance and mechanisms of burst release in matrix-controlled drug delivery systems. J Control Release, 2001. 73(2-3): 121-36.

22. Lee J and Lee K.Y. Local and sustained vascular endothelial growth factor delivery for angiogenesis using an injectable system. Pharm Res, 2009. 26(7): 1739-44.

23. Jeon $\mathrm{O}$, et al. Long-term and zero-order release of basic fibroblast growth factor from heparin-conjugated poly(L-lactide-co-glycolide) nanospheres and fibrin gel. Biomaterials, 2006. 27(8): 1598-607.
24. Kim M.S, et al. Development of functional fibrous matrices for the controlled release of basic fibroblast growth factor to improve therapeutic angiogenesis. Tissue Eng Part A, 2010. 16(10): 2999-3010.

25. Pike D.B, et al. Heparin-regulated release of growth factors in vitro and angiogenic response in vivo to implanted hyaluronan hydrogels containing VEGF and bFGF. Biomaterials, 2006. 27(30): 5242-51.

26. Ruvinov E, Leor J, and Cohen S. The effects of controlled HGF delivery from an affinity-binding alginate biomaterial on angiogenesis and blood perfusion in a hindlimb ischemia model. Biomaterials, 2010. 31(16): 4573-82.

27. Fujita $\mathrm{M}$, et al. Therapeutic angiogenesis induced by controlled release of fibroblast growth factor- 2 from injectable chitosan/non-anticoagulant heparin hydrogel in a rat hindlimb ischemia model. Wound Repair Regen, 2007. 15(1): 58-65.

28. DuBose J.W, Cutshall C, and Metters A.T. Controlled release of tethered molecules via engineered hydrogel degradation: model development and validation. J Biomed Mater Res A, 2005. 74(1): 104-16.

29. Ehrbar M, et al. Cell-demanded liberation of VEGF121 from fibrin implants induces local and controlled blood vessel growth. Circ Res, 2004. 94(8): 1124-32.

30. Sun $Q$, et al. Sustained release of multiple growth factors from injectable polymeric system as a novel therapeutic approach towards angiogenesis. Pharm Res, 2010. 27(2): 264-71.

31. Layman H, et al. Co-delivery of FGF-2 and G-CSF from gelatin-based hydrogels as angiogenic therapy in a murine critical limb ischemic model. Acta Biomater, 2009. 5(1): 230-9.

32. Patil S.D, Papadmitrakopoulos F, and Burgess D.J. Concurrent delivery of dexamethasone and VEGF for localized inflammation control and angiogenesis. J Control Release, 2007. 117(1): 68-79.

33. Peattie R.A, et al. Dual growth factor-induced angiogenesis in vivo using hyaluronan hydrogel implants. Biomaterials, 2006. 27(9): 1868-75.

34. Tengood J.E, et al. Sequential delivery of vascular endothelial growth factor and sphingosine 1-phosphate for angiogenesis. Biomaterials, 2010. 31(30): 7805-12

35. Sun G, et al. Functional neovascularization of biodegradable dextran hydrogels with multiple angiogenic growth factors. Biomaterials, 2011. 32(1): 95-106.

36. Silva E.A and Mooney D.J. Effects of VEGF temporal and spatial presentation on angiogenesis. Biomaterials, 2010. 31(6): 1235-41.

37. Tang D.W, et al. Heparinized chitosan/poly(gamma-glutamic acid) nanoparticles for multi-functional delivery of fibroblast growth factor and heparin. Biomaterials, 2010. 31(35): 9320-32.

38. Garbern J.C, et al. Delivery of basic fibroblast growth factor with a $\mathrm{pH}$-responsive, injectable hydrogel to improve angiogenesis in infarcted myocardium. Biomaterials, 2011. 32(9): 2407-16.

39. Yu J, et al. The effect of injected RGD modified alginate on angiogenesis and left ventricular function in a chronic rat infarct model. Biomaterials, 2009. 30(5): 751-6.

40. Chen S.Y, et al. Autologous transplantation of EPCs encoding FGF1 gene promotes neovascularization in a porcine model of chronic myocardial ischemia. Int J Cardiol, 2009. 135(2): 223-32.

41. Duan H.F, et al. Treatment of myocardial ischemia with bone marrow-derived mesenchymal stem cells overexpressing hepatocyte growth factor. Mol Ther, 2003. 8(3): 467-74.

42. Iwaguro $H$, et al. Endothelial progenitor cell vascular endothelial growth factor gene transfer for vascular regeneration. Circulation, 2002. 105(6): 732-8.

43. Matsumoto $\mathrm{R}$, et al. Vascular endothelial growth factor-expressing mesenchymal stem cell transplantation for the treatment of acute myocardial infarction. Arterioscler Thromb Vasc Biol, 2005. 25(6): 1168-73.

44. Sun L, et al. Mesenchymal stem cells modified with angiopoietin-1 improve remodeling in a rat model of acute myocardial infarction. Biochem Biophys Res Commun, 2007. 357(3): 779-84.

45. Cheng $Z$, et al. Targeted migration of mesenchymal stem cells modified with CXCR4 gene to infarcted myocardium improves cardiac performance. Mol Ther, 2008. 16(3): 571-9.

46. Jabbarzadeh E, et al. Induction of angiogenesis in tissue-engineered scaffolds designed for bone repair: a combined gene therapy-cell transplantation approach. Proc Natl Acad Sci U S A, 2008. 105(32): 11099-104.

47. Tang J, et al. Mesenchymal stem cells over-expressing SDF-1 promote angiogenesis and improve heart function in experimental myocardial infarction in rats. Eur J Cardiothorac Surg, 2009. 36(4): 644-50.

48. Tang J, et al. Combination of chemokine and angiogenic factor genes and mesenchymal stem cells could enhance angiogenesis and improve cardiac function after acute myocardial infarction in rats. Mol Cell Biochem, 2010. 339(1-2): 107-18. 
49. Zhang D, et al. Over-expression of CXCR4 on mesenchymal stem cells augments myoangiogenesis in the infarcted myocardium. J Mol Cell Cardiol, 2008. 44(2): 281-92.

50. Zhu X.Y, et al. Transplantation of adipose-derived stem cells overexpressing hHGF into cardiac tissue. Biochem Biophys Res Commun, 2009. 379(4): 1084-90.

51. Park H.J, Yang F, and Cho S.W. Nonviral delivery of genetic medicine for therapeutic angiogenesis. Adv Drug Deliv Rev, 2012. 64(1): 40-52.

52. Das $\mathrm{H}$, et al. Stem cell therapy with overexpressed VEGF and PDGF genes improves cardiac function in a rat infarct model. PLoS One, 2009. 4(10): e7325.

53. Kim S.H, et al. Hypoxia-inducible vascular endothelial growth factor-engineered mesenchymal stem cells prevent myocardial ischemic injury. Mol Ther, 2011. 19(4): 741-50.

54. Yang F, et al. Genetic engineering of human stem cells for enhanced angiogenesis using biodegradable polymeric nanoparticles. Proc Natl Acad Sci U S A, 2010. 107(8): 3317-22.

55. Mangi A.A, et al. Mesenchymal stem cells modified with Akt prevent remodeling and restore performance of infarcted hearts. Nat Med, 2003. 9(9): 1195-201.

56. Shujia J, et al. Stable therapeutic effects of mesenchymal stem cell-based multiple gene delivery for cardiac repair. Cardiovasc Res, 2008. 77(3): 525-33.

57. Ceradini D.J, et al. Progenitor cell trafficking is regulated by hypoxic gradients through HIF-1 induction of SDF-1. Nat Med, 2004. 10(8): 858-64.

58. Dimmeler S. Regulation of bone marrow-derived vascular progenitor cell mobilization and maintenance. Arterioscler Thromb Vasc Biol, 2010. 30(6): 1088-93.

59. Chen F.M, et al. Homing of endogenous stem/progenitor cells for in situ tissue regeneration: Promises, strategies, and translational perspectives. Biomaterials, 2011. 32(12): 3189-209.

60. Petit I, et al. G-CSF induces stem cell mobilization by decreasing bone marrow SDF-1 and up-regulating CXCR4. Nat Immunol, 2002. 3(7): 687-94.

61. Pitchford S.C, et al. Differential mobilization of subsets of progenitor cells from the bone marrow. Cell Stem Cell, 2009. 4(1): 62-72.

62. Orlic D, et al. Mobilized bone marrow cells repair the infarcted heart, improving function and survival. Proc Natl Acad Sci U S A, 2001. 98(18): 10344-9.

63. Zaruba M.M, et al. Synergy between CD26/DPP-IV inhibition and G-CSF improves cardiac function after acute myocardial infarction. Cell Stem Cell, 2009. 4(4): 313-23. 